\title{
Rectal cancer patients can be treated conservatively
}

\author{
Krzysztof Bujko
}

Introduction. The strategy of undertaking a watch-and-wait policy without surgical treatment is currently being debated in those patients where complete clinical response/regression (cCR) has been diagnosed after preoperative irradiation. Its proponents maintain that there is sufficient evidence of efficacy allowing routine use. This means that following preoperative chemoradiotherapy, each patient should be investigated for CCR. If so diagnosed, physicians are then obliged to give the patient the choice of two treatment options: surgical treatment or watch-and-wait without surgery. Radical surgical treatment is adopted as a rescue/salvage therapy if during the watch-and-wait period, local recurrence in patients occurs. In contrast, opponents of the strategy of watch-and-wait, purport that evidence for its efficacy is inadequate to justify routine use.

Results. Literature data indicates that the rate of $\mathrm{CCR}$ depends on the tumour size, with incidence ranging between $5 \%$ and $78 \%$ of those patients irradiated prior to surgery. If the patients were kept under watch-and-wait without surgery, then the local recurrence rate was around 30\%. Such high rates of local recurrences does not however disqualify this approach because the efficacy of rescue surgery was high. Distant metastases were rare, up $10 \%$ of patients, and survivals were even better compared to control patient groups who had been operated on due to not achieving CCR; this difference arising from the inherently less aggressive tumours that are sensitive to irradiation.

Conclusions. The data hitherto indicate that a policy of watch-and-wait may be adopted in patients with CCR after chemoradiotherapy. Nevertheless, introducing this strategy requires acquiring experience for diagnosing CCR and an efficient organisation and supervision of treatment along with the rigorous follow-up.

NOWOTWORY J Oncol 2017; 67, 2: 146-151

Key words: rectal cancer, preoperative radiotherapy, watch-and-wait policy

\section{Introduction}

Adopting a watch-and-wait policy in patients diagnosed with complete clinical response (cCR) following preoperative irradiation, is the most 'hot topic' being currently debated in rectal cancer; taking place both in the literature $[1,2]$ and at scientific conferences. The essence of this debate can be succinctly summarised as follows: Those advocating watch-and-wait for CCR patients uphold that evidence for its efficacy is sufficient for adopting it to routine treatment [1]. In concrete terms, they argue that anyone with pre-operative chemoradiotherapy should be screened for CCR. Whenever diagnosed, the physician is obliged to present the patient with two possible options: surgical treatment such as previously planned, or a watch-and-wait approach without surgery. Radical surgical treatment is then used during follow-up only as a rescue procedure in patients with local recurrence. Proponents of watch-and-wait emphasise the advantages of this approach; where the anus and whole rectum are preserved. This provides better anorectal and sexual functions compared to surgery, and thus a better quality of life. In addition, there is no mortality and other postoperative complications. Contrastingly, the opponents of a watch-and-wait policy argue that there is insufficient evidence for the effectiveness of this method in the literature for routine use [2]. They argue that this issue still requires further research before it can be used in routine practice. This viewpoint is shared by most physicians, as reflected in all the known official guidelines. 
In preoperative radiotherapy, the watch-and-wait policy gives rise to a paradox. On the one hand, radiotherapy is discontinued in patients with small cancers for two reasons: 1) After using modern-day surgery, i.e. total mesorectal excision, rates of local recurrence are very low, and 2) Radiation therapy results in complications, which is most commonly an increase in the anterior resection syndrome, i.e. anorectal dysfunctions. On the other hand, proponents of watchand-wait maintain that indications for patient irradiation with small cancers should be widened, as these patients are more likely to develop cCR than in advanced cancer cases. This allows organ preservation if CCR is achieved after chemoradiotherapy, which thereby provides better anorectal function compared to surgically treated patients [3].

Despite many studies, prior to treatment, no test has yet been developed that could predict a pathological complete response ( $\mathrm{pCRc}$ ) with sufficient accuracy. It is therefore not possible to decide before treatment whether only chemoradiotherapy is sufficient, or whether resection becomes necessary.

\section{Biological backgrounds \\ for a watch-and-wait policy \\ Relationship between radiationsensitivity and aggressiveness of rectal cancer}

Rectal cancer is a heterogeneous disease. About 15\% of all cancer are radiosensitive, which after preoperative chemoradiotherapy and resection causes that no cancer is found by the pathologist in postoperative specimen, either in the bowel or regional lymph nodes. In patients attaining $\mathrm{pCR}$, prognosis is significantly better than in those without pCR. For example, Mass et al. found $16 \%$ of pCR patients from all preoperative chemoradiotherapy in a multiple centres study [4]. At fFive years local recurrence was observed in $3 \%$ of patients and distant metastases in $9 \%$, whilst in those with persisting cancer after chemoradiotherapy such rates were respectively $10 \%$ and $23 \%$.

Five-year cancer-free survival was $83 \%$ in $\mathrm{pCR}$ patients and $66 \%$ in patients with persistent cancer; $p<0.001$. This indicates that radiosensitive cancer is less aggressive than radioresistant cancer. This is not only reflected in lowered local recurrence rates, but also by a reduced susceptibility to distant metastases [4].

\section{Efficacy of rescue surgery}

Local recurrences after $\mathrm{CCR}$ are common, being about one third of patients [5-8]. This is opposed to around 5\% relapses occurring after surgery. For this reason, an integral part of the watch-and-wait policy is rescue surgery in the event of local recurrence. A condition for adopting this approach, as a valuable treatment, is the high efficacy of rescue surgery. Indeed, hitherto published long-term outcomes after rescue surgery do not appear any worse than those for patients operated on directly after chemoradiotherapy when having the same stages of cancer $[5,6]$. It should however be stressed that that these studies were often performed on a limited number of patients, and observations were frequently short-term.

The most numerous study by Habr-Gama et al. [5], include only 28 patients with local recurrences occurring during observation in $\mathrm{CCR}$ patients after receiving chemoradiotherapy. In 5 of these (18\%), distant metastases were observed in further follow-up. Rescue surgery was performed in 26 patients, 4 of whom had local recurrence. Thus, $79 \%$ of patients with locally advanced disease were finally cured of the primary tumour.

Likewise, favourable outcomes have been reported in other studies on less numerous patient groups and with shorter follow-ups [6-9]. These showed significantly better efficacy than those obtained after treatment of local recurrence after radical surgery. In such patients, effective radical resection of the recurrence is rarely possible due to involvement of other organs and/or the coexistence of distant metastases. If the resection succeeds, long-term outcomes are however unfavourable. Because of the substantially different outcomes after the treating these two types of recurrence, one study group has proposed that this term be reserved only for local recurrence after radical surgical treatment, whereas local recurrence occurring in patients during watch-and-wait should be termed 'regrowth' [1].

For the same reason, another study did not consider regrowth when undergoing radical rescue surgery as a failure of treatment [7]. Treatment failure was recognised only when there was recurrent local re-regrowth after rescue surgery or when distant metastases were diagnosed.

\section{Correlation between $C C R$ and $p C R$}

CCR does not always mean that there are no residual, residual subclinical disease in the bowel wall or lymph nodes of the mesorectum. This has been shown by pathological studies on patients that underwent resection despite achieving CCR. In around $30 \%$ of these cases, cancer cells were found in the bowel wall $[10,11]$. As mentioned previously, in a similar number of cases, regrowths were seen in the bowel wall if patients had been under observation without surgery [5-8]. In addition, in all operated patients with bowel wall cPR, 5\% were observed to have mesorectal lymph node metastases. Indeed, in those patients observed after $\mathrm{CCR}$, there were only a few cases of recurrence found in the mesorectal nodes [6]. Nevertheless, this kind of regrow occurs rarely; recurrence in the bowel wall is much more frequent.

Likewise, when $\mathrm{pCR}$ is recognised this does not necessarily mean that so is $\mathrm{CCR}$. In a significant proportion of $\mathrm{pCR}$ cases, there is a persistent tumour or ulcer present [10-14]. Only microscopic examination shows that the tumour is not malignant, but is only composed of stromal fibrous tissue. 
Table I. Clinical and pathological complete cancer response to preoperative chemoradiotherapy according to tumour progression

\begin{tabular}{lccccc}
\hline Tumour size / Study author & $\begin{array}{c}\text { Number of } \\
\text { patients }\end{array}$ & CCR (\%) & pCR (\%) & $\begin{array}{c}\text { CCR patients (\%) } \\
\text { not achieving pCRPR }\end{array}$ & $\begin{array}{c}\text { pCRCPR patients (\%) } \\
\text { not achieving CCR }\end{array}$ \\
\hline $\begin{array}{l}\text { Small tumours up to 3 cm, } \\
\text { mainly T2 }\end{array}$ & & & & & \\
$\quad$ Garcia-Anguilar [10] & 77 & $56 \%$ & $44 \%$ & $33 \%$ & $15 \%$ \\
$\quad$ Bujko [11] & 89 & $34 \%$ & $44 \%$ & $38 \%$ & $33 \%$ \\
Advanced tumours T3-4 & & & & & $86 \%$ \\
$\quad$ Grillem [13] & 94 & $3 \%$ & $15 \%$ & $8 \%$ & $62 \%$ \\
$\quad$ Smith F [14] & 220 & $6 \%$ & $14 \%$ & & \\
\hline
\end{tabular}

cCR — clinical complete regression; $\mathrm{PCRCPR}$ - pathological complete regression

Table I presents outcomes of prospective studies evaluating clinical and pathological rates of complete responses to preoperative chemoradiotherapy in relation to tumour size $[10,11,13,14]$. The smaller the tumour, the more often is pCR achieved after chemoradiotherapy and the more often does it coincide with cCR [10-12]. Preoperative radiotherapy in patients with advanced cancer results in about $15 \%$ of $\operatorname{pCR}[4,6,14]$. However, cCR occurs less frequently in around $5 \%$ of patients [15].

In most patients with a large tumour prior to treatment and in whom $\mathrm{pCR}$ was achieved, there was however no cCR as the remaining tumour consisting of solely fibrous tissue imitating persistent cancer [13-15]. In patients with up to a $3 \mathrm{~cm}$ diameter cancer, $\mathrm{CCR}$ and $\mathrm{pCR}$ rates increase to about $45 \%[10,11]$ and around $2 / 3$ rds of patients achieving $C C R$ are also diagnosed with $\mathrm{pCR}$; in the remaining $1 / 3 \mathrm{rd}$, despite $C C R$, microscopic disease is observed upon histopathological examination (clinical assessment being false positive).

Table I shows that there are two main criteria determining the best candidates for undergoing watch-and-wait, namely those with a small size cancer but not those with large tumours; pCR being more often achieved which more frequently correlates with $\mathrm{CCR}$.

Besides the studies presented in Table I, others confirm the above conclusions. A study by Das et al. [16] on 108 patients achieving $\mathrm{pCR}$ after chemoradiotherapy demonstrated a $\mathrm{pCR}$ rate of $23 \%$ in those with tumours involving less than $60 \%$ of the bowel circumference, compared to $13 \%$ in tumour occupied more than $60 \%$ of the bowel circumference.

Multivariate analysis has showed that the extent to which the bowel circumference is involved constitutes the only factor associated with pCR. Van Stiphout et al. [17], studied 953 patients, and demonstrated that the length of the intestinal wall involved, significantly correlated with the $\mathrm{pCR}$ rates. The included nomogram shows that the $\mathrm{pCR}$ rate clearly increases when the tumour is less than $5 \mathrm{~cm}$ in size.

PCR and CCR rates are not dependent on whether clinically diagnosed metastases to regional lymph nodes are present. Complete regression at the primary tumour was achieved in a similar proportion of patients without clinically diagnosed nodal metastases, like in patients with metastases $[6,9,18]$. Furthermore, there was a strong correlation between the radiosensitiveness of the primary tumour with the radiosensitiveness of the metastases to lymph nodes. Thus, if a complete regression of the primary tumour was achieved, it was most commonly accompanied by complete regression of nodal metastases. As aforementioned, the proportion of patients with ypTON+ disease was only $5 \%$ of all patients with ypT0 [4].

\section{Long-term results in CCR patients undergoing watch-and-wait}

Table II presents prospective and retrospective study outcomes evaluating efficacy when surgical intervention is withheld if CCR is achieved 6-10 weeks after chemoradiotherapy. This data was retrieved following a systematic review of the literature. Outcomes of most studies are encouraging. A watch-and-wait policy was only tested in two randomised trials [15, 19]; design of one of these studies having been methodologically optimal [15]. Patients achieving CCR after chemoradiotherapy were randomly selected for watch-and-wait or total mesorectal excision. Only 6 patients (5\%) out of 160 achieved cCR over two years of recruitment; two were randomized for resection (the pathological study revealed ypTONO and ypT2N0) and four for watch-and-wait (one was found to have regrowth whilst the other three had sustained cCR).

For the second randomised study, patients were qualified ( $n=88$ ) with a low-lying tumour with CT2 or CT3 disease, involving no more than $2 / 3$ rds of the bowel circumference [19]. Both arms of this study used preoperative radiotherapy; in one, a dose of $39 \mathrm{~Gy}$ in 17 fractions was given, whilst in the second the dose was increased with ortovoltage irradiation and in patients achieving complete regression, brachytherapy was also given. In the study arm, using the increased irradiation dose, 6 patients (14\%) achieved cCR. These patients underwent watch-and-wait without surgery; none of them had any local recurrence during a long followup period. In both randomised groups, the proportions of 
Table II. Prospective studies in patients with clinical complete cancer regression (CCR) after chemoradiotherapy undergoing observation without surgery

\begin{tabular}{|c|c|c|c|}
\hline Study author & $\begin{array}{l}\text { Number of patients } \\
\text { treated by irradiation }\end{array}$ & $\begin{array}{l}\text { CCR rate }(\%) \text { in patients } \\
\text { without surgery }\end{array}$ & $\begin{array}{l}\text { Local recurrence rates (\%) in cCR patients, } \\
\text { median follow-up }\end{array}$ \\
\hline \multicolumn{4}{|c|}{ Habr-Gama Team } \\
\hline Habr-Gama [23] & 173 & $33 \%$ & $11 \%$ in patients achieving cCR after 12 months 65 months. \\
\hline Habr-Gama [21] & 361 & $\begin{array}{l}34 \% \text {, including ypT0 patients } \\
\text { after local excision }\end{array}$ & $24 \%$ (19\% during first year \& $5 \%$ later), 60 months \\
\hline Habr-Gama [22] & 29 & $48 \%$ & $21 \%, 23$ months \\
\hline Habr-Gama [5] & 183 & $49 \%$ & $31 \%, 60$ months \\
\hline \multicolumn{4}{|l|}{ Other authors } \\
\hline Martens [6] & Numbers unknown & 100 patients & $15 \%, 41$ months \\
\hline Appelt [8] & 51 & $78 \%$ & $16 \%$ after 1 year observation \\
\hline Renehan [7] & 259 & $12 \%+98$ registered patients & $34 \%, 33$ months \\
\hline Dalton [23] & 49 & $24 \%$ & $50 \%, 25$ months \\
\hline Nahas [15] & 118 & $5 \%$ & 1 amongst 4 \\
\hline Araujo [27] & Numbers unknown & 42 & $28 \%, 48$ months \\
\hline Yu [26] & Numbers unknown & $\begin{array}{l}19 \mathrm{cCR} \text { patients and small } \\
\text { residual tumour }\end{array}$ & $47 \%$, observation - no data \\
\hline Rupinski [28] & 35 & $31 \%$ & Observation too short for assessment \\
\hline Nakagawa [25] & 52 & $19 \%$ & $80 \%$ \\
\hline Smith [9] & 297 & Unknown, $11 \%$ cCR observed & $19 \%, 23$ months \\
\hline Hughes [24] & 266 & Unknown, 4\% cCR observed & $60 \%, 46$ months \\
\hline Ortholan [19] & 42 & $14 \%$ & $0 \%, 132$ months \\
\hline
\end{tabular}

local recurrences, overall survival and disease-free survival were similar.

All other studies were observational. The pioneer for propounding the watch-and-wait policy is Habr-Gama's team from Brazil [5, 18, 20-22]. In those patients where achieving $\mathrm{CCR}$ is uncertain, then a full-thickness local excision of the tumour is performed. It is noteworthy that the primary tumour in patients treated by this team was not large, where the mean tumour size in one study was $3.7 \mathrm{~cm}$ and in many patients did not penetrate outside of the bowel wall (cT2) [5]. In one study, cCR was achieved in $49 \%$ of patients [5] in whom local recurrence was observed in 31\% of cases. Outcomes of rescue surgery in these patients are described above. In the whole group of patients achieving cCR, cancer specific survival was $94 \%$ after 5 years.

Renehan et al. [7] reported the routine use of watch-andwait in $12 \%$ of patients who achieved cCR after routine preoperative chemoradiotherapy for consecutive patients from three UK centres. Although local recurrence was in $34 \%$ of patients, the overall survival after 3 years was $96 \%$ compared with $87 \%$ in control group that underwent surgery after chemoradiotherapy; $p=0.024$. It should be deemed that the inferior survival in the group of surgical patients arose from biologically inherent lower aggressiveness of radiosensitive cancers; this issue is presented in the text above.

In a prospective study on 51 patients, Appelt et al. [8] showed that unlike most published reports, where routine preoperative chemoradiotherapy was used with 45-50 Gy, the dose here was increased to $60 \mathrm{~Gy}$ with external beams. In addition, 5 Gy was administered to one brachytherapy fraction by an applicator placed in the rectum; the proportion achieving $\mathrm{CCR}$ being as high as $78 \%$. These patients underwent watch-and-wait without surgery. The risk of local recurrence after one year was $16 \%$. All reports indicate that distant metastases in CCR patients are rare, being at a maximum of $10 \%$ patients. Outcomes from other studies are presented in Table II [23-27]. In several of these, the decision not to treat metastases in regional lymph nodes $(\mathrm{cN}+)$ was not a contraindication for watch-and-wait $[6,9,18]$. It has not been shown that treatment outcomes in this subgroup were any worse than patients diagnosed as CNO before treatment.

At our centre a prospective study was conducted on patients aged over 70 years with tumours of up to $5 \mathrm{~cm}$ involving no more than $60 \%$ of the bowel wall circumference [28]. The main justification for this study was in the chances for improving survival in patients achieving $\mathrm{CCR}$ resulting from the avoidance of postoperative mortality [29]. Postoperative mortality after total mesorectal excision in patients older than 75 years was high, and was $16 \%$ after 6 months compared to $4 \%$ in younger patients [30]. Of the first 35 study patients, CCR was achieved in 31\% of cases [29]. CCR was observed not only after chemoradiotherapy but also in 4 out of 14 patients (29\%) irradiated with $5 \times 5$ Gy and 
then evaluated after about 10 weeks. A $5 \times 5$ Gy treatment was administered to patients unfit for chemotherapy. CCR patients were observed. In addition, $34 \%$ of the patients underwent local resection of the residual tumour. Thus in total, organ preservation was achieved in $66 \%$ of patients. The too short observations made it impossible to evaluate long-term outcomes.

The Table II data show large differences both in the achieved CCR rates and local recurrence rates in patients without surgery. This could have been caused by selecting patients with very advanced cancer as candidates for conservative treatment or through using a wide definition of cCR. For example, Yu et al. [26] and Hughes et al. [24] reported high rates of local recurrence, which did not include endoscopic evaluation and was based solely on MR or on digital rectal examinations.

\section{Definition of CCR}

Recently, Habr-Gama et al. [31] have defined cCR in detail. According to her, cCR should only be diagnosed if there are no mucosal lesions or if a smooth scar with or without telangiectasia is visible; mucosal stiffening is possible at this location. Any ulcer, even superficial ones, palpable lumps or mucosal irregularities and bowel lumen narrowing are treated as being non-cCR. Retrospective photographic image analyses of postoperative material showed that if this definition was used, only $10 \%$ of patients with a pathological diagnosis of complete regression coincidences with the clinical diagnosis of complete regression [14].

It should be stressed that none of the imaging investigations such as CT, MRI or PET are enough accurate that they can be used to determine whether $\mathrm{pCR}$ was also achieved in CCR patients [32-34]. In the latter, pelvic MRI is rather an auxiliary aid, since diagnosing residual lesions does not exclude the diagnosis of complete regression [31]. This issueis described in detail by Lambregts et al. [32]. MRI serves rather as a baseline investigation, possibly needed for later comparisons during subsequent follow-up. Similarly, histopathological examination of scar or persisting lesions biopsies after radiotherapy is not reliable because of the high rate of false-negatives $[28,35,36]$. Residual cancer is difficult to detect, as single cancer cells survived in the deeper layers of the submucosal or in muscularis propia $[37,38]$.

\section{Conclusions}

Hitherto, data indicates that a watch-and-wait strategy may be used in patients achieving CCR after chemoradiotherapy. Introducing this approach nevertheless requires that experience in diagnosing $\mathrm{CCR}$ be acquired, along with efficient organisation and supervision over treatment and follow-up.

Conflict of interest: none declared

\section{Prof. Krzysztof Bujko, MD, PhD}

Department of Radiotherapy

The Maria Skłodowska-Curie Memorial Cancer Centre

and Institute of Oncology

W.K. Roentgena 5

02-781 Warszawa, Poland

e-mail:bujko@coi.waw.pl

\section{Received \& Accepted: 4 Oct 2016}

Based on the presentation at the IV Annual Conference of the Nowotwory Journal of Oncology, 'Oncological Debates', held in Warszawa, 8-9th April 2016

\section{References}

1. Heald RJ, Beets G, Carvalho C. Report from a consensus meeting: response to chemoradiotherapy in rectal cancer - predictor of cure and a crucial new choice for the patient: on behalf of the Champalimaud 2014 Faculty for 'Rectal cancer: when NOT to operate'. Colorectal Dis 2014; 16: 334-337.

2. Marijnen CA. Organ preservation in rectal cancer: have all questions been answered? Lancet Oncol 2015; 16: e13-22.

3. Habr-Gama A, Lynn PB, Jorge JM et al. Impact of organ-preserving strategies on anorectal function in patients with distal rectal cancer following neoadjuvant chemoradiation. Dis Colon Rectum 2016; 59: 264-269.

4. Maas M, Nelemans PJ, Valentini V et al. Long-term outcome in patients with a pathological complete response after chemoradiation for rectal cancer: a pooled analysis of individual patient data. Lancet Oncol 2010; 11:835-844.

5. Habr-Gama A, Gama-Rodrigues J, Sao Julia GP et al. Local recurrence after complete clinical response and watch and wait in rectal cancer after neoadjuvant chemoradiation: impact of salvage therapy on local disease control. Int J Radiat Oncol Biol Phys 2014; 88: 822-828.

6. Martens $\mathrm{MH}, \mathrm{Maas} \mathrm{M}$, Heijnen LA et al. Long-term outcome of an organ preservation program after neoadjuvant treatment for rectal cancer. J Natl Cancer Inst 2016; 108(12) Pll: djw 171.

7. Renehan AG, Malcomson L, Emsley R et al. Watch-and-wait approach versus surgical resection after chemoradiotherapy for patients with rectal cancer (the OnCoRe project): a propensity-score matched cohort analysis. Lancet Oncol 2016; 17: 174-183.

8. Appelt AL, Pløen J, Harling $\mathrm{H}$ et al. High-dose chemoradiotherapy and watchful waiting for distal rectal cancer: a prospective observational study. Lancet Oncol 2015; 16: 919-927.

9. Smith JD, Ruby JA, Goodman KA et al. Nonoperative management of rectal cancer with complete clinical response after neoadjuvant therapy. Ann Surg 2012; 256: 965-972.

10. Garcia-Aguilar J, Shi Q, Thomas CR Jr et al. A phase II trial of neoadjuvant chemoradiation and local excision for T2NO rectal cancer: preliminary results of the ACOSOG Z6041 trial. Ann Surg Oncol 2012; 19: 384-391.

11. Bujko K, Richter P, Smith FM et al. Preoperative radiotherapy and local excision of rectal cancer with immediate radical re-operation for poor responders: a prospective multicentre study. Radiother Oncol 2013; 106: 198-205.

12. Gérard JP, Chamorey E, Gourgou-Bourgade $\mathrm{S}$ et al. Clinical complete response ( $\mathrm{CCR}$ ) after neoadjuvant chemoradiotherapy and conservative treatment in rectal cancer. Findings from the ACCORD 12/PRODIGE 2 randomized trial. Radiother Oncol 2015; 115: 246-252.

13. Guillem JG, Chessin DB, Shia J et al. Clinical examination following preoperative chemoradiation for rectal cancer is not a reliable surrogate end point. J Clin Oncol 2005; 23: 3475-3479.

14. Smith FM, Chang KH, Sheahan $\mathrm{K}$ et al. The surgical significance of residual mucosal abnormalities in rectal cancer following neoadjuvant chemoradiotherapy. Br J Surg 2012; 99: 993-1001.

15. Nahas SC, Rizkallah Nahas CS, Sparapan Marques CF et al. Pathologic complete response in rectal cancer: can we detect it? Lessons learned from a proposed randomized trial of watch-and-wait treatment of rectal cancer. Dis Colon Rectum. 2016; 59: 255-263.

16. Das $\mathrm{P}$, Skibber JM, Rodriguez-Bigas MA et al. Predictors of tumor response and downstaging in patients who receive preoperative chemoradiation for rectal cancer. Cancer 2007; 109: 1750-1755. 
17. van Stiphout RG, Lammering G, Buijsen J et al. Development and external validation of a predictive model for pathological complete response of rectal cancer patients including sequential PET-CT imaging. Radiother Oncol 2011; 98: 126-133.

18. Habr-Gama A, Perez RO, Proscurshim I et al. J. Patterns of failure and survival for nonoperative treatment of stage c0 distal rectal cancer following neoadjuvant chemoradiation therapy. J Gastrointest Surg 2006; 10: 1319-1328; discussion 1328-1329.

19. Ortholan $C$, Romestaing $\mathrm{P}, \mathrm{Chapet} \mathrm{O}$ et al. Correlation in rectal cancer between clinical tumor response after neoadjuvant radiotherapy and sphincter or organ preservation: 10-year results of the Lyon R 96-02 randomized trial. Int J Radiat Oncol Biol Phys 2012; 83: e165-171.

20. Habr-Gama A, Perez RO, Nadalin W et al. Long-term results of preoperative chemoradiation for distal rectal cancer correlation between final stage and survival. J Gastrointest Surg 2005; 9: 90-99.

21. Habr-Gama A, Perez RO, Sabbaga J et al. Increasing the rates of complete response to neoadjuvant chemoradiotherapy for distal rectal cancer: results of a prospective study using additional chemotherapy during the resting period. Dis Colon Rectum 2009; 52: 1927-1934.

22. Habr-Gama A, Perez RO, Sao Juliao GP et al. Nonoperative approaches to rectal cancer: a critical evaluation. Semin Radiat Oncol 2011; 21: 234-239.

23. Dalton RS, Velineni R, Osborne ME et al. A single-centre experience of chemoradiotherapy for rectal cancer: is there potential for nonoperative management? Colorectal Dis 2012; 14: 567-571.

24. Hughes R, Harrison M, Glynne-Jones R. Could a wait and see policy be justified in T3/4 rectal cancers after chemo-radiotherapy? Acta Oncol 2010; 49: 378-381.

25. Nakagawa WT, Rossi BM, de O Ferreira F et al. Chemoradiation instead of surgery to treat mid and low rectal tumors: is it safe? Ann Surg Oncol 2002; 9: 568-573.

26. Yu SK, Brown G, Heald RJ et al. Deferral of rectal surgery following a continued response to preoperative chemoradiotherapy (watch and wait) study: A phase II multicenter study in the United Kingdom. J Clin Oncol 2011; 29 (Suppl 4; abstr 489).

27. Araujo RO, Valadão $M$, Borges $D$ et al. Nonoperative management of rectal cancer after chemoradiation opposed to resection after complete clinical response. A comparative study. Eur J Surg Oncol 2015; 41: 1456-1463.
28. Rupinski M, Szczepkowski M, Malinowska M et al. Watch and wait policy after preoperative radiotherapy for rectal cancer; management of residual lesions that appear clinically benign. Eur J Surg Oncol 2016; 42: 288-296.

29. Smith FM, Rao C, Oliva Perez R et al. Avoiding radical surgery improves early survival in elderly patients with rectal cancer, demonstrating complete clinical response after neoadjuvant therapy: results of a decision-analytic model. Dis Colon Rectum 2015; 58: 159-171.

30. Rutten $\mathrm{H}$, den Dulk $\mathrm{M}$, Lemmens $\mathrm{V}$ et al. Survival of elderly rectal cancer patients not improved: analysis of population based data on the impact of TME surgery. Eur J Cancer 2007; 43: 2295-2300.

31. Habr-Gama A, Perez RO, Wynn G et al. Complete clinical response after neoadjuvant chemoradiation therapy for distal rectal cancer: characterization of clinical and endoscopic findings for standardization. Dis Colon Rectum 2010; 53: 1692-1698.

32. Lambregts DM, Maas M, Bakers FC et al. Long-term follow-up features on rectal MRI during a wait-and-see approach after a clinical complete response in patients with rectal cancer treated with chemoradiotherapy. Dis Colon Rectum 2011; 54: 1521-1528.

33. Perez RO, Habr-Gama A, Gama-Rodrigues J et al. Accuracy of positron emission tomography/computed tomography and clinical assessment in the detection of complete rectal tumor regression after neoadjuvant chemoradiation: Long-term results of a prospective trial (National Clinical Trial 00254683). Cancer 2012; 118: 3501-3511.

34. Guillem JG, Ruby JA, Leibold T et al. Neither FDG-PET nor CT is able to distinguish between a pathological complete response and an incomplete response after neoadjuvant chemoradiation in locally advanced rectal cancer: a prospective study. Ann Surg 2013; 258: 289-295.

35. Perez RO, Habr-Gama A, Pereira GV et al. Role of biopsies in patients with residual rectal cancer following neoadjuvant chemoradiation after downsizing: can they rule out persisting cancer? Colorectal Dis 2012; 14: 714-720.

36. Maretto I, Pomerri F, Pucciarelli S et al. The potential of restaging in the prediction of pathologic response after preoperative chemoradiotherapy for rectal cancer. Ann Surg Oncol 2007; 14: 455-461.

37. Duldulao MP, Lee W, Streja $L$ et al. Distribution of residual cancer cells in the bowel wall after neoadjuvant chemoradiation in patients with rectal cancer. Dis Colon Rectum 2013; 56: 142-149.

38. Hayden DM, Jakate S, Pinzon MC et al. Tumor scatter after neoadjuvant therapy for rectal cancer: are we dealing with an invisible margin? Dis Colon Rectum 2012; 55: 1206-1212. 